\title{
Flaw Tolerance in a Viscoelastic Strip
}

e-mail: chenshaohua72@hotmail.com

LNM,

Institute of Mechanics,

Chinese Academy of Sciences,

Beijing 100190, China

\author{
Huajian Gao ${ }^{1}$ \\ School of Engineering, \\ Brown University, \\ Providence, RI 02912 \\ e-mail: Huajian_Gao@brown.edu
}

Load-bearing biological materials such as bone, teeth, and nacre have acquired some interesting mechanical properties through evolution, one of which is the tolerance of cracklike flaws incurred during tissue function, growth, repair, and remodeling. While numerous studies in the literature have addressed flaw tolerance in elastic structures, so far there has been little investigation of this issue in time-dependent, viscoelastic systems, in spite of its importance to biological materials. In this paper, we investigate flaw tolerance in a viscoelastic strip under tension and derive the conditions under which a pre-existing center crack, irrespective of its size, will not grow before the material fails under uniform rupture. The analysis is based on the Griffith and cohesive zone models of crack growth in a viscoelastic material, taking into account the effects of the loading rate along with the fracture energy, Young's modulus, and theoretical strength of material. [DOI: 10.1115/1.4007864]

Keywords: biological materials, flaw tolerance, fracture mechanics, viscoelasticity, loading rate

\section{Introduction}

It has been well established that micro- and nano-structured materials can exhibit drastically different properties compared to their macroscopic counterparts. For example, while an ideal defect-free solid could, in principle, attain its theoretical strength irrespective of the sample size, the load-carrying capacity of a macroscopic material is always compromised by the inevitable existence of cracklike flaws. As a result, the actual strength of the material is usually size-dependent and can only approach theoretical strength when the sample size is reduced to nanoscale.

The notion that biological systems have achieved flaw tolerance via size reduction and organized structural hierarchy has been extensively discussed in the literature [1-8]. A structure is said to be flaw tolerant if pre-existing flaws do not propagate until it ultimately fails through a uniform rupture near the theoretical strength of the material. This concept can be related to many classical studies in fracture mechanics on notch insensitivity, fracture size effects, large scale yielding, and bridging [9-23] and has been particularly helpful in understanding the hierarchical structures of bonelike materials and biological adhesive systems [1,3-5,7,8,24-32]. It has been shown that biological materials/systems tend to fail by uniform rupture, rather than by crack propagation, due to the selection of characteristic sizes in their hierarchical structures.

Gao and Chen [6] investigated the conditions under which an elastic strip becomes flaw tolerant. The basic length scale of flaw tolerance is defined as $l_{\mathrm{ft}}=\Gamma E / \sigma_{s}^{2}$, where $\Gamma$ is the fracture energy, $E$ is the Young's modulus, and $\sigma_{s}$ is the theoretical strength of the material. According to Gao and Chen [6], the Griffith model of crack growth leads to the following critical structural size for flaw tolerance

$$
\left\{\begin{array}{l}
\frac{W_{\mathrm{ft}}}{l_{\mathrm{ft}}}=\min _{0 \leq \beta<1}\left(\frac{\kappa}{\pi \beta(1-\beta)^{2} F^{2}(\beta)}\right) \\
F(\beta)=\left(1-0.025 \beta^{2}+0.06 \beta^{4}\right) \sqrt{\sec \left(\frac{\pi \beta}{2}\right)}, \quad \beta=\frac{a}{W}
\end{array}\right.
$$

\footnotetext{
${ }^{1}$ Corresponding authors.

Manuscript received June 21, 2012; final manuscript received September 25, 2012; accepted manuscript posted October 22, 2012; published online May 16, 2013. Assoc. Editor: Anand Jagota.
}

where $W$ is the half-width of the strip; $a$ is the half-length of a pre-existing center crack, so that $\beta=a / W$ measures an effective area density of the crack over the cross-section of the strip; here, $\kappa=1 /\left(1-\nu^{2}\right)$ for the plane strain and $\kappa=1$ for the plane stress. In comparison, the corresponding critical size, according to the Dugdale-Barenblatt model, is [6]

$$
\left\{\begin{array}{l}
\frac{W_{\mathrm{ft}}}{l_{\mathrm{ft}}}=\min _{0 \leq \beta<1}\left(\frac{\pi \kappa}{4 f(\beta)}\right) \\
f(\beta)=\int_{\beta}^{1} \ln \frac{\sin \pi(\xi+\beta) / 2}{\sin \pi(\xi-\beta) / 2} d \xi
\end{array}\right.
$$

Recently, Kumar et al. [33,34] performed in situ TEM experiments of the tensile fracture in thin aluminum strips with a preexisting edge notch and observed that the samples sometimes fail away from the notch and there was no measurable stress concentration near the notch tip. Other experiments showed that silk fibril bundles with diameters in the range of $20-150 \mathrm{~nm}$ can be scaled up to form macroscopic silk fibers with outstanding mechanical properties in spite of the presence of cavities, tears, and cracks [35].

The mechanical properties of protein-rich biomaterials such as bone are usually time-dependent [36,37] and viscoelastic or poroelastic models are often important for biomedical applications such as developing materials to match the time-dependent behaviors of bone for viscoelastic biocompatibility [38]. In fact, almost all solid biomaterials or tissues exhibit viscoelastic behaviors to some extent. The reader can be referred to Fung's book [39] for numerous examples.

In the present paper, we investigate the flaw tolerance condition of a center-cracked viscoelastic strip under tension, deriving analytical solutions based on the Griffith and Dugdale-Barenblatt models of crack growth in terms of the loading rate and material constants such as the fracture energy, Young's modulus, and theoretical strength.

\section{Model}

The model under consideration involves a viscoelastic strip of width $2 W$ containing an interior center crack of length $2 a$ subject to a uniaxial, time-dependent tensile loading $\sigma_{\infty} f_{\sigma}(t)$, as shown in Fig. 1, where $f_{\sigma}(t)$ is a function representing a time-dependent loading profile. 


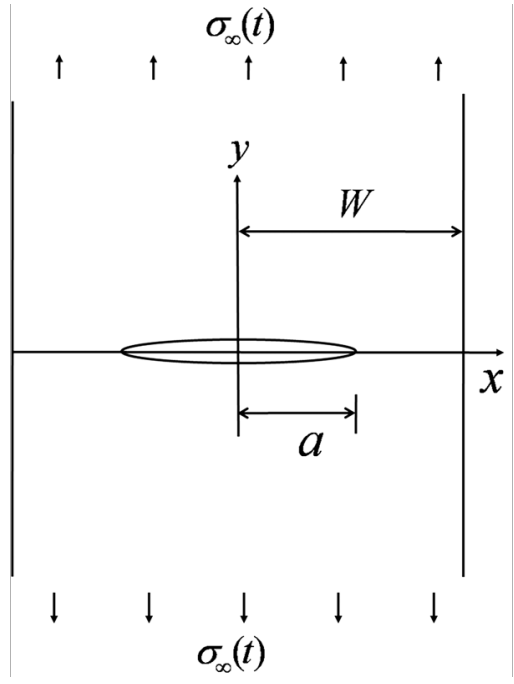

Fig. 1 A center-cracked viscoelastic strip of width $2 W$ and crack size $2 a$ under tension

The strip is assumed to obey the standard linear model of viscoelasticity (see Fig. 2), with the following uniaxial constitutive relation

$$
\sigma+p_{1} \dot{\sigma}=q_{0} \varepsilon+q_{1} \dot{\varepsilon}
$$

where

$$
p_{1}=\frac{\eta}{E_{1}+E_{2}}, \quad q_{0}=\frac{E_{1} E_{2}}{E_{1}+E_{2}}, \quad q_{1}=\frac{E_{1} \eta}{E_{1}+E_{2}}, \quad q_{1}>p_{1} q_{0}
$$

with $E_{1}, E_{2}$, and $\eta$ being the spring and dashpot constants shown in Fig. 2. The solution to Eq. (3) can be expressed in an integral form as

$$
\varepsilon(t)=J(t)^{*} d \sigma(t)=\sigma(0) J(t)+\int_{0^{+}}^{t} J(t-\tau) \frac{\partial \sigma(\tau)}{\partial \tau} d \tau
$$

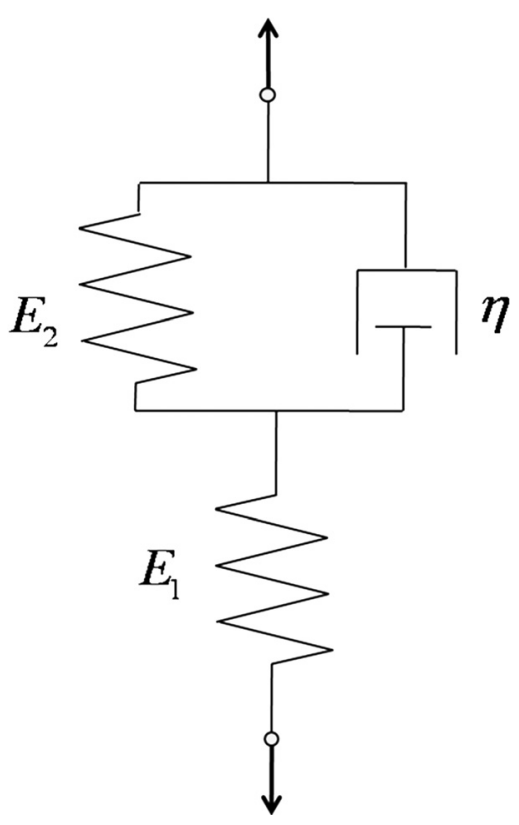

Fig. 2 The standard viscoelastic model used in the present study

$$
\sigma(t)=E(t)^{*} d \varepsilon(t)=\varepsilon(0) E(t)+\int_{0^{+}}^{t} E(t-\tau) \frac{\partial \varepsilon(\tau)}{\partial \tau} d \tau
$$

where ${ }^{*} d$ denotes the Stieltjes convolution and $J(t)$ and $E(t)$ are creep compliance and stress relaxation functions

$$
\begin{gathered}
J(t)=\frac{1}{q_{0}}-\frac{q_{1}-p_{1} q_{0}}{q_{1} q_{0}} e^{-q_{0} t / q_{1}}=J_{\infty}-\left(J_{\infty}-J_{0}\right) e^{-t / \tau_{r}} \\
E(t)=q_{0}+\left(\frac{q_{1}}{p_{1}}-q_{0}\right) e^{-t / p_{1}}=E_{\infty}-\left(E_{\infty}-E_{0}\right) e^{-t / \tau_{0}}
\end{gathered}
$$

In the preceding expressions, $E_{0}$ and $E_{\infty}$ are the instantaneous and long-time elastic moduli, $J_{0}$ and $J_{\infty}$ are the instantaneous and long-time compliances, $\tau_{0}$ is the relaxation time, and $\tau_{r}$ is the retardation time. One can easily verify the following relations

$$
\begin{gathered}
E_{0}=1 / J_{0}=E_{1} \\
E_{\infty}=1 / J_{\infty}=E_{1} E_{2} /\left(E_{1}+E_{2}\right) \\
\tau_{0}=p_{1}=E_{\infty} \tau_{r} / E_{0}=\eta /\left(E_{1}+E_{2}\right)
\end{gathered}
$$

For simplicity, Poisson's ratio of the viscoelastic strip is taken here as a time-independent constant.

\section{Flaw Tolerance Analysis Based on Griffith Model of Crack Growth}

3.1 Auxiliary Elastic Model. We first consider the auxiliary problem of an elastic strip of width $2 W$ containing a central crack of length $2 a$, as shown in Fig. 3(a). The corresponding viscoelastic problem is shown in Fig. 3(b). In the auxiliary elastic model, $\sigma^{e}$ denotes the applied uniaxial stress and $\sigma_{y}^{e}(x, 0), \varepsilon_{y}^{e}(x, 0)$, and $u_{y}^{e}(x, 0)$ denote the normal stress, strain, and displacement along the crack plane, respectively. In the viscoelastic model, $\sigma^{v}(t)$ denotes the applied load and $\sigma_{y}^{v}(x, 0, t), \varepsilon_{y}^{v}(x, 0, t)$, and $u_{y}^{v}(x, 0, t)$ are the normal stress, strain, and displacement along the crack plane, respectively.

The two models satisfy the following relations

$$
\sigma^{v}(t)=\sigma^{e} f_{\sigma}(t)=\sigma_{\infty} f_{\sigma}(t)
$$

between the applied loads and we have

$$
\begin{array}{r}
\sigma_{y}^{v}(x, 0, t)=\sigma_{y}^{e}(x, 0) f_{\sigma}(t) \\
u_{y}^{v}(x, 0, t)=u_{y}^{e}(x, 0) f_{u}(t) \\
\varepsilon_{y}^{v}(x, 0, t)=\varepsilon_{y}^{e}(x, 0) f_{u}(t)
\end{array}
$$

along the crack plane, where $f_{\sigma}(t)$ and $f_{u}(t)$ are the time-dependent parts of the stress and displacement, respectively.

In fracture mechanics, the energy release rates for the elastic and viscoelastic models can be written in the form

$$
G_{I}^{e}=\lim _{\Delta a \rightarrow 0} \frac{1}{\Delta a} \int_{0}^{\Delta a} \sigma_{y}^{e}(x, 0) u_{y}^{e}(x, 0) d x=\frac{K_{I}^{e 2}}{\kappa E^{e}}
$$

and

$$
G_{I}^{v}(t)=\lim _{\Delta a(t) \rightarrow 0} \frac{1}{\Delta a(t)} \int_{0}^{\Delta a(t)} \sigma_{y}^{v}(x, 0, t) u_{y}^{v}(x, 0, t) d x
$$

where $E^{e}$ is the Young's modulus in the auxiliary elastic problem, which can be chosen arbitrarily; here, $\kappa=1 /\left(1-\nu^{2}\right)$ for the plane strain and $\kappa=1$ for the plane stress and $K_{I}^{e}=\sigma_{\infty} \sqrt{\pi a} F(\beta)$ denotes the elastic stress intensity factor under the external loading $\sigma_{\infty}$, where $F(\beta)$ is defined in Eq. (1). The energy release rate 


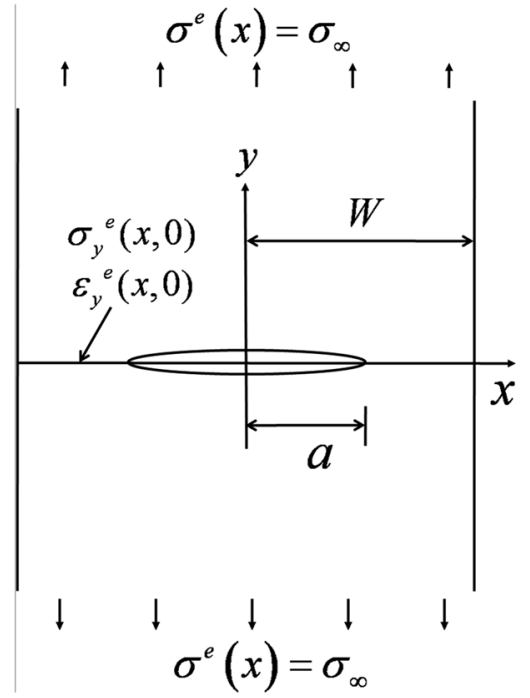

(a)

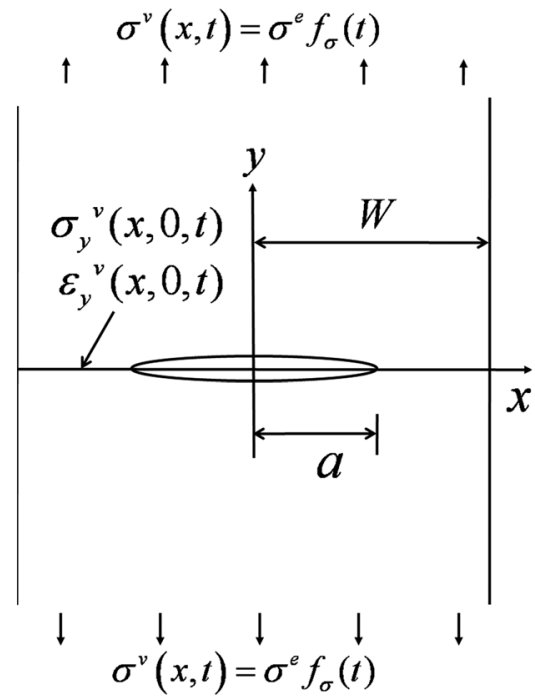

(b)

Fig. 3 Analysis of the viscoelastic strip problem with Griffith's model of crack growth via the correspondence principle. (a) The auxiliary problem of an elastic strip of width $2 W$ and crack size $2 a$, and $(b)$ the corresponding viscoelastic problem.

associated with crack initiation in the viscoelastic material is calculated by substituting Eqs. (10a)-(10c) into Eq. (12). This leads to [40]

$G_{I}^{v}(t)=\lim _{\Delta a \rightarrow 0} \int_{0}^{\Delta a} \frac{1}{\Delta a}\left[\sigma_{y}^{e}(x, 0) f_{\sigma}(t)\right]\left[u_{y}^{e}(x, 0) f_{u}(t)\right] d x=G_{I}^{e} f_{\sigma}(t) f_{u}(t)$

where $G_{I}^{e}$ denotes the energy release rate from the corresponding elastic problem in Eq. (11). The functions $f_{u}(t)$ and $f_{\sigma}(t)$ will be determined from the correspondence principle between the viscoelastic and elastic boundary value problems $[41,42]$.

3.2 Energy Release Rate for Crack Initiation in Viscoelastic Material. Applying the Laplace transform to the stress, strain, and displacement functions in Eqs. $(10 a)-(10 c)$ and the constitutive relation Eq. (3) leads to

$$
\begin{aligned}
& \bar{\sigma}_{y}(x, 0, s)=\sigma_{y}^{e}(x, 0) \bar{f}_{\sigma}(s) \\
& \bar{\varepsilon}_{y}(x, 0, s)=\varepsilon_{y}^{e}(x, 0) \bar{f}_{u}(s) \\
& \bar{u}_{y}(x, 0, s)=u_{y}^{e}(x, 0) \bar{f}_{u}(s) \\
& \bar{\sigma}\left(1+p_{1} s\right)=\bar{\varepsilon}\left(q_{0}+q_{1} s\right)
\end{aligned}
$$

Here, an overbar on a variable denotes its Laplace transform. Equation (17) corresponds to the transformed uniaxial stressstrain relation.

For a mode I crack, the stress state ahead of the crack tip is equi-biaxial, i.e., $\sigma_{y}^{e}=\sigma_{x}^{e}$. In this state, the stress-strain relation could be written as

$$
\varepsilon_{y}^{e}=\frac{\rho}{E^{e}} \sigma_{y}^{e}
$$

where $\rho=\left(1-v-2 v^{2}\right)$ under plane strain and $\rho=(1-v)$ under plane stress.

After the Laplace transform, Eq. (18) becomes

$$
\bar{\varepsilon}_{y}=\frac{\left(1+p_{1} s\right)}{\left(q_{0}+q_{1} s\right)} \rho \bar{\sigma}_{y}
$$

Substituting Eqs. (14) into (19) leads to

$$
\bar{\varepsilon}_{y}(x, 0, s)=\rho \sigma_{y}^{e}(x, 0) \frac{\bar{f}_{\sigma}(s)\left(1+p_{1} s\right)}{\left(q_{0}+q_{1} s\right)}
$$

which, after an inverse Laplace transform, results in

$$
\varepsilon_{y}^{v}(x, 0, t)=\rho \sigma_{y}^{e}(x, 0) \mathrm{L}^{-1}\left[\frac{\bar{f}_{\sigma}(s)\left(1+p_{1} s\right)}{\left(q_{0}+q_{1} s\right)}\right]
$$

Here, $L^{-1}[\ldots]$ denotes the inverse Laplace transform of the bracketed term.

Combining Eqs. (10c), (18), and (21) yields the following relation

$$
f_{u}(t)=E^{e} \mathbf{L}^{-1}\left[\frac{\bar{f}_{\sigma}(s)\left(1+p_{1} s\right)}{\left(q_{0}+q_{1} s\right)}\right]
$$

The time-dependent energy release rate can be obtained from Eqs. (13) and (22) as

$$
G_{I}(t)=G_{I}^{e} f_{\sigma}(t) E^{e} \mathbf{L}^{-1}\left[\frac{\bar{f}_{\sigma}(s)\left(1+p_{1} s\right)}{\left(q_{0}+q_{1} s\right)}\right]
$$

Substituting Eqs. (11) into (23), we have

$$
G_{I}(t)=\frac{\left(K_{I}^{e}\right)^{2} f_{\sigma}(t)}{\kappa} \mathrm{L}^{-1}\left[\frac{\bar{f}_{\sigma}(s)\left(1+p_{1} s\right)}{\left(q_{0}+q_{1} s\right)}\right]
$$

3.3 Flaw Tolerant Analysis With Time-Dependent Energy Release Rate. For the center-cracked viscoelastic strip to be flaw tolerant, the energy release rate should not exceed the fracture energy of the material (assumed to be a material constant), i.e.,

$$
G_{I}(t) \leq \Gamma
$$

before the strip fails at its theoretical strength $(1-\beta) \sigma_{s}$, regardless of the crack size.

This condition is expressed as

$$
\frac{\left[(1-\beta) \sigma_{s} \sqrt{\pi a} F(\beta)\right]^{2} f_{\sigma}(t)}{\kappa} \mathrm{L}^{-1}\left[\frac{\bar{f}_{\sigma}(s)\left(1+p_{1} s\right)}{\left(q_{0}+q_{1} s\right)}\right] \leq \Gamma
$$


For a given $\beta$ and loading time $t_{0}$ at which the external loading reaches the theoretical strength of the strip, Eq. (26) gives rise to a critical strip width $W_{\mathrm{cr}}$ from $G_{I}(t)=\Gamma$ as

$$
W_{\mathrm{cr}}=\left.\frac{\Gamma \kappa}{\pi \beta(1-\beta)^{2} F^{2}(\beta) \sigma_{s}^{2} f_{\sigma}(t) \mathrm{L}^{-1}\left[\frac{\bar{f}_{\sigma}(s)\left(1+p_{1} s\right)}{\left(q_{0}+q_{1} s\right)}\right]}\right|_{t=t_{0}}, \quad(0 \leq \beta<1)
$$

The flaw tolerant width $W_{\mathrm{ft}}$ is then defined as

$$
W_{\mathrm{ft}}=\min _{0 \leq \beta<1}\left(W_{\mathrm{cr}}\right)
$$

Introducing an intrinsic length scale [6]

$$
l_{\mathrm{ft}}=\frac{\Gamma E_{\infty}}{\sigma_{s}^{2}}
$$

the flaw tolerant size can be normalized as

$$
\frac{W_{\mathrm{ft}}}{l_{\mathrm{ft}}}=\left.\min _{0 \leq \beta<1}\left(\frac{\kappa}{\pi \beta(1-\beta)^{2} F^{2}(\beta)}\right) \frac{1}{f_{\sigma}(t) \mathrm{L}^{-1}\left[\frac{\bar{f}_{\sigma}(s)\left(1+p_{1} s\right)}{\left(1+q_{1} s / q_{0}\right)}\right]}\right|_{t=t_{0}}
$$

In the preceding equation, the time independent part within the round bracket on the right side is identical to the solution for the corresponding elastic problem [6]; the remaining part represents the effects of the viscoelastic parameters of the strip and the loading rate.

It follows that the flaw tolerant size of a viscoelastic strip is

$$
W_{\mathrm{ft}}=\left.\frac{\chi \Gamma E_{\infty}}{\sigma_{s}^{2}} \frac{1}{f_{\sigma}(t) \mathrm{L}^{-1}\left[\frac{\bar{f}_{\sigma}(s)\left(1+p_{1} s\right)}{\left(1+q_{1} s / q_{0}\right)}\right]}\right|_{t=t_{0}}
$$

where $\chi=\min _{0 \leq \beta<1}\left(\kappa / \pi \beta(1-\beta)^{2} F^{2}(\beta)\right)$. Taking $\nu=0.3$ yields $\chi=2.1$ for the plane strain and $\chi=1.91$ for the plane stress.

3.4 A Simple Example Case. As a simple example, consider a bi-linear loading profile to the theoretical strength $\sigma_{\infty}=(1-\beta) \sigma_{s}$, as shown in Fig. 4. The applied loading function

$$
\sigma=\sigma_{\infty} f_{\sigma}(t)=\sigma_{\infty}\left[\frac{t}{t_{0}}-\frac{\left(t-t_{0}\right) H\left(t-t_{0}\right)}{t_{0}}\right], \quad 0 \leq t<\infty
$$

leads to the following displacement function

$$
f_{u}(t)=E^{e} \frac{t+\left(t_{0}-t\right) H\left(t-t_{0}\right)+\left[H\left(t-t_{0}\right)+1\right]\left(q_{1}-p_{1} q_{0}\right)\left(1+e^{\left[-q_{0}\left(t-t_{0}\right) / q_{1}\right]}\right)}{q_{0} t_{0}}
$$

where $H(t)$ denotes the Heaviside function: $H(t)=0$ for $t<0$ and $H(t)=1$ for $t \geq 0$.

For viscoelastic materials such as butyl rubber, we take the material constants as $E_{1}=115 \mathrm{MPa}, E_{2}=130 \mathrm{MPa}$, $\eta=0.49 \mathrm{MPas}, \Gamma=0.01 \mathrm{~J} / \mathrm{m}^{2}$, and $\sigma_{s}=0.61 \mathrm{MPa}$ [43], which results in $\tau_{0}=2 \mathrm{~ms}$. The stress-strain relations for the butyl rubber under different loading rates are shown in Fig. 5, from which one can see that the stiffness of the viscoelastic material increases with the loading rate $\tau_{0} / t_{0}$. At very low loading rates, the viscoelastic rubber behaves like an elastic material with a long-time modulus $E_{\infty}$, while it assumes the instantaneous modulus $E_{0}$ at very high loading rates. Figure 6 shows the relationship between the normalized critical strip width for crack growth versus the relative crack size under different loading rates and plane stress conditions. The flaw tolerance width, corresponding to the minimum strip width below which cracks of all sizes are tolerated, is seen to increase with the loading rate. This is consistent with the fact that a higher loading rate corresponds to a larger Young's modulus; hence, a larger flaw tolerant size.

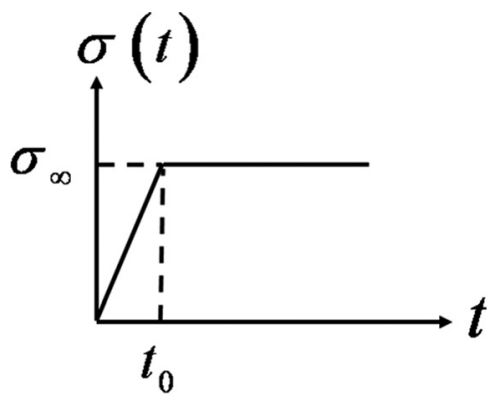

Fig. 4 A bi-linear loading profile in the viscoelastic problem

\section{Flaw Tolerant Analysis Based on Dugdale Model}

4.1 Elastic Solution. Consider the elastic model shown in Fig. 7, where an elastic strip of half-width $W$ containing a center crack of length $2 a$ is subject to an applied uniaxial stress $\sigma_{\infty}$. The cohesive zone ahead of the crack is $l$, leading to an effective crack length of $2 c$ with a crack tip opening displacement $\delta$ and

$$
c=a+l
$$

Within the cohesive zone, the normal traction $\sigma(\delta)$ is related to the effective range of cohesive interaction $\delta_{c}$ as follows

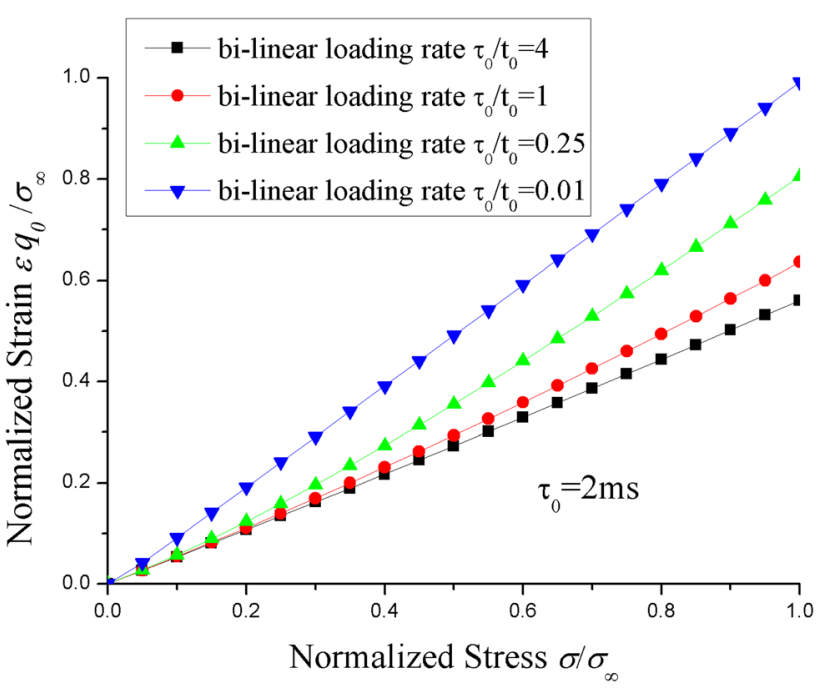

Fig. 5 The stress-strain relationship of a viscoelastic butyl rubber under different loading rates $\tau_{0} / t_{0}$ 


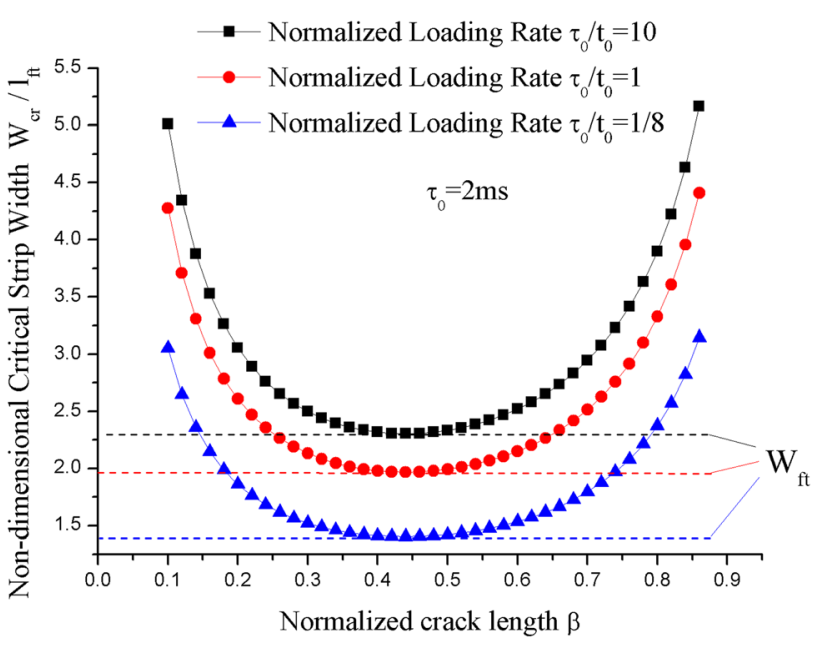

Fig. 6 The normalized critical strip width $W_{\mathrm{cr}} / I_{\mathrm{ft}}$ for crack growth as a function of the normalized crack length $\beta=a / W$ in the viscoelastic Griffith problem under different loading rates

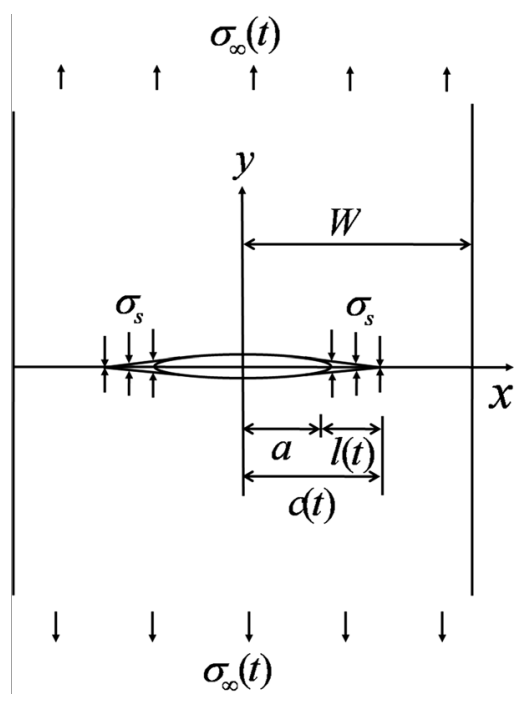

Fig. 7 A center-cracked elastic strip of width $2 W$ subject to remote tension $\sigma_{\infty}$. The length of the cohesive zone is $I$ and the effective crack length is $c=a+l$.

$$
\sigma(\delta)= \begin{cases}\sigma_{s}, & 0<\delta \leq \delta_{c} \\ 0, & \delta>\delta_{c}\end{cases}
$$

The flaw tolerance solution corresponds to a uniform distribution of the normal stress $\sigma_{s}$ outside the crack region. To find the crack tip opening displacement, the original model is divided into three subproblems, as shown in Fig. 8: (1) a perfect crack-free strip subjected to uniform stress $\sigma_{\infty}$, as shown in Fig. 8(a), (2) a center-cracked strip with crack length $2 c$ subject to a uniform normal compressive stress $\sigma_{\infty}$ on the crack face, as shown in Fig. 8(b), and (3) a uniform normal tensile stress $\sigma_{s}$ in the cohesive zone of length $l$, as shown in Fig. 8(c). Since there is no contribution to the crack opening displacement from the first subproblem, the total crack opening displacement from the other two subproblems can be found from the classical distributed dislocation density method as (see the Appendix)

$$
\delta=\frac{8 \sigma_{s} W}{\pi \kappa E^{e}} I(\alpha)
$$

where

$I(\alpha)=\int_{\theta}^{\pi / 2} \frac{c^{\prime} \cos X}{\pi \sqrt{1-c^{\prime 2} \sin ^{2} X}} \ln \frac{(\sin \theta-\sin X)(1-\cos (\theta+X))}{(\sin \theta+\sin X)(1-\cos (\theta-X))} d X$

Here, $\sin \theta=a^{\prime} / c^{\prime}, \quad a^{\prime}=\sin (\pi \beta / 2)$, and $c^{\prime}=\sin (\pi \alpha / 2)$. The normalized effective crack length $\alpha=c / W$ is deduced in the Appendix as

$$
\alpha=\frac{c}{W}=\frac{2}{\pi} \arcsin \frac{\sin \frac{\pi \beta}{2}}{\cos \frac{\pi \sigma_{\infty}}{2 \sigma_{s}}}
$$

Using the preceding crack opening displacement, the flaw tolerant condition for the elastic strip requires

$$
\sigma_{\infty}=(1-\beta) \sigma_{s}, \quad \alpha=1
$$

and

$$
\delta=\left.\frac{8 \sigma_{s} W}{\pi \kappa E^{e}} I(\alpha)\right|_{\alpha=1} \leq \delta_{c}=\frac{\Gamma}{\sigma_{s}}
$$

The flaw tolerant width is thus

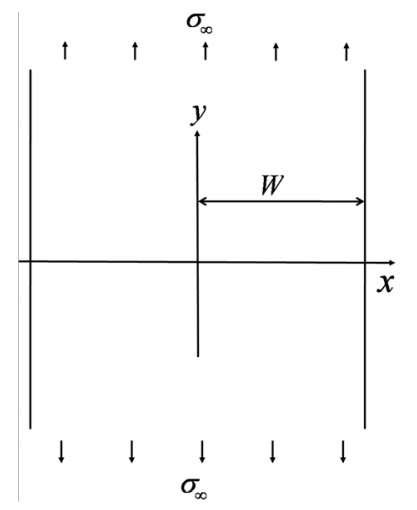

(a)

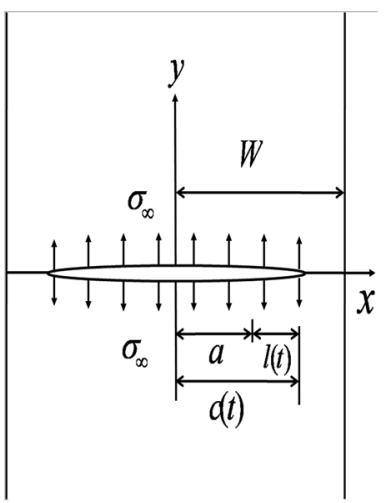

(b)

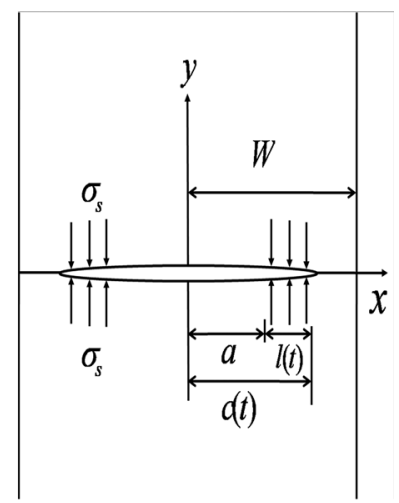

(c)

Fig. 8 Superposition scheme used to determine the crack opening displacement in the elastic Dugdale problem. (a) A perfect strip without crack under applied stress $\sigma_{\infty}$; a strip with crack length $2 c$ subjected to $(b)$ a uniformly normal compressive stress $\sigma_{\infty}$ on the crack face, and $(c)$ a uniformly normal tensile stress $\sigma_{s}$ in the cohesive zone. 


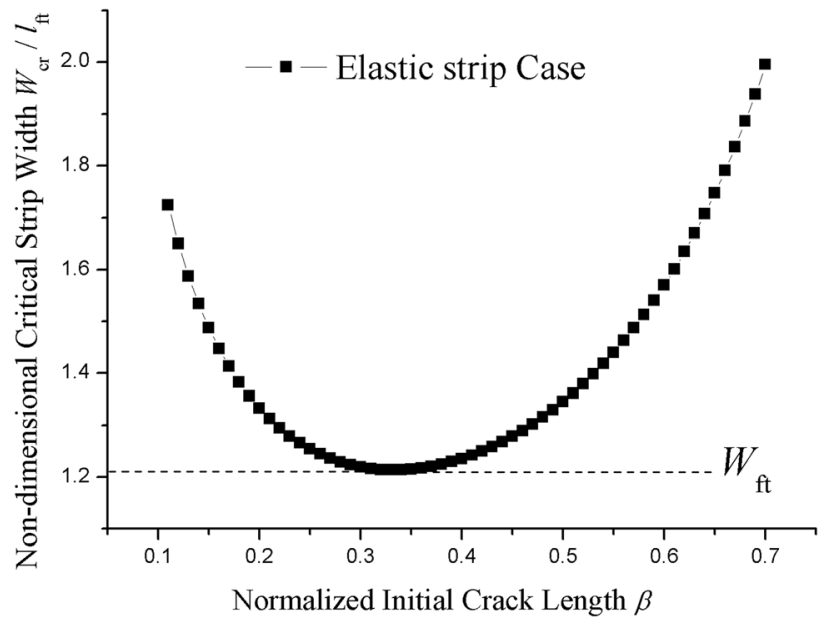

Fig. 9 The normalized critical strip width $W_{\mathrm{cr}} / I_{\mathrm{ft}}$ for crack growth as a function of the normalized crack length $\beta=a / W$ in the elastic Dugdale problem

$$
W_{\mathrm{ft}}=\min _{0 \leq \beta<1}\left(W_{\mathrm{cr}}\right)=\min _{0 \leq \beta<1}\left(\frac{\delta_{c} \pi \kappa E^{e}}{\left.8 \sigma_{s} I(\alpha)\right|_{\alpha=1}}\right)
$$

below which, cracks of all sizes are tolerated by the strip. The normalized flaw tolerant width $W_{\mathrm{ft}}$ is defined as

$$
\frac{W_{\mathrm{ft}}}{l_{\mathrm{ft}}}=\min _{0 \leq \beta<1}\left(\frac{\pi \kappa}{\left.8 I(\alpha)\right|_{\alpha=1}}\right)
$$

where $l_{\mathrm{ft}}=\Gamma E^{e} / \sigma_{s}^{2}=\delta_{c} E^{e} / \sigma_{s}$ is the previously mentioned intrinsic length scale of flaw tolerance.

Figure 9 shows the normalized critical width for crack growth $W_{\mathrm{cr}} / l_{\mathrm{ft}}$ versus the relative crack size $\beta$. The minimum value of $W_{\text {cr }} / l_{\mathrm{ft}}$ yields $W_{\mathrm{ft}} / l_{\mathrm{ft}}$, which is consistent with the earlier result given by Gao and Chen [6].
4.2 Viscoelastic Solution. Graham's extended correspondence principle $[44,45]$ can be used to determine the viscoelastic solution of the crack opening displacement based on the Dugdale model. This principle, when applied to a moving crack, states that the stress distribution in quasi-static plane problems is the same for elastic and viscoelastic solids under the conditions that: (1) the crack size does not decrease $(d c / d t \geq 0)$, (2) the elastic stress normal to the plane of crack prolongation is independent of elastic constants, and (3) any dependence of the normal displacement along the crack face on elastic constants can be written in the separation form $u_{y}=f_{1}(E, \nu) f_{2}(x)$.

Consider a center-cracked viscoelastic strip subject to a uniaxial tensile loading

$$
\sigma(x, t)=\sigma_{\infty} f_{\sigma}(t)
$$

where $f_{\sigma}(t)$ is a monotonically increasing function and $0 \leq f_{\sigma}(t) \leq 1$. It can be shown that all three conditions of Graham's extended correspondence principle are satisfied in the present model.

Replacing the external loading $\sigma_{\infty}$ in the elastic solution to the effective crack length $\alpha=c / W$ by the time-dependent loading in the viscoelastic model, we obtain the time-dependent effective crack length directly from Eq. (37)

$$
\alpha(t)=\frac{c(t)}{W}=\frac{2}{\pi} \arcsin \frac{\sin \frac{\pi \beta}{2}}{\cos \frac{\pi \sigma_{\infty} f_{\sigma}(t)}{2 \sigma_{s}}}
$$

Applying the extended correspondence principle to Eq. (36) yields the time-dependent crack opening displacement in the viscoelastic model as

$$
\delta(t)=\frac{8 W \sigma_{s}}{\pi \kappa}\left[J(t) I(c(0))+\int_{0^{+}}^{t} J^{\prime}(t-\tau) \frac{\partial I(c(\tau))}{\partial \tau} d \tau\right]
$$

where

$$
I(c(t))=\int_{\theta(t)}^{\pi / 2} \frac{c^{\prime}(t) \cos X}{\pi \sqrt{1-\left[c^{\prime}(t)\right]^{2} \sin ^{2} X}} \ln \frac{[\sin \theta(t)-\sin X]\{1-\cos [\theta(t)+X]\}}{[\sin \theta(t)+\sin X]\{1-\cos [\theta(t)-X]\}} d X
$$

Here, $\sin \theta(t)=a^{\prime} / c^{\prime}(t), a^{\prime}=\sin (\pi \beta / 2)$, and $c^{\prime}(t)=\sin (\pi \alpha(t) / 2)$.

4.3 Flaw Tolerance Analysis Based on the Dugdale Model. Since the ultimate load carrying capacity of the viscoelastic strip is $(1-\beta) \sigma_{s}$, the load on the remote boundary is taken to be

$$
\sigma=\sigma_{\infty} f_{\sigma}(t)=(1-\beta) \sigma_{s} f_{\sigma}(t)
$$

In the state of flaw tolerance, the crack tip opening displacement remains below the effective interaction range $\delta_{c}$, i.e.,

$$
\delta(t)=\frac{8 W \sigma_{s}}{\pi \kappa}\left[J(t) I(c(0))+\int_{0^{+}}^{t} J^{\prime}(t-\tau) \frac{\partial I(c(\tau))}{\partial \tau} d \tau\right] \leq \delta_{c}
$$

Here, $\delta_{c}$ is assumed to be a material constant.

For a given $\beta$ and loading time $t_{0}$, there is a critical strip width $W_{\text {cr }}$ for the crack growth, i.e., $\delta(t)=\delta_{c}$

$$
\begin{gathered}
W_{\mathrm{cr}}=\left.\frac{\pi \kappa \delta_{c}}{8 \sigma_{s}\left[J\left(t_{0}\right) I(c(0))+\int_{0^{+}}^{t_{0}} J^{\prime}\left(t_{0}-\tau\right) \frac{\partial I(c(\tau))}{\partial \tau} d \tau\right]}\right|_{t=t_{0}} \\
\quad(0 \leq \beta<1)
\end{gathered}
$$

The flaw tolerant width $W_{\mathrm{ft}}$ is then

$$
\begin{aligned}
\frac{W_{\mathrm{ft}}}{l_{\mathrm{ft}}} & =\min _{0 \leq \beta<1}\left(\frac{W_{\mathrm{cr}}}{l_{\mathrm{ft}}}\right) \\
& =\left.\min _{0 \leq \beta<1}\left\{\frac{\pi \kappa}{8\left[\tilde{J}\left(t_{0}\right) I(c(0))+\int_{0^{+}}^{t_{0}} \tilde{J}^{\prime}\left(t_{0}-\tau\right) \frac{\partial I(c(\tau))}{\partial \tau} d \tau\right]}\right\}\right|_{t=t_{0}}
\end{aligned}
$$

where $l_{\mathrm{ft}}=\delta_{c} E_{\infty} / \sigma_{s}$ is the intrinsic length [6] and $\tilde{J}$ is the normalized creep compliance defined as

$$
\tilde{J}=E_{\infty} J
$$




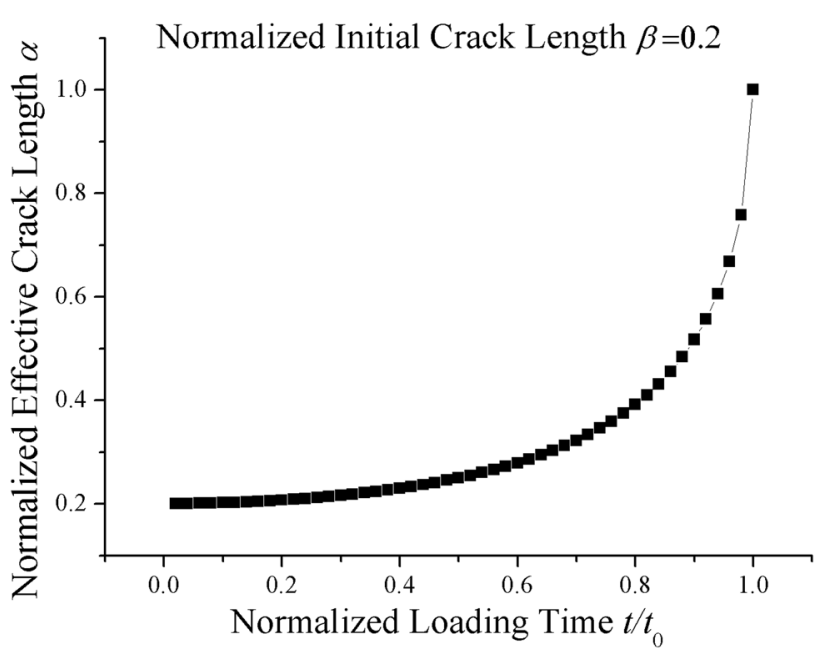

Fig. 10 The normalized effective crack length as a function of the loading time for the viscoelastic problem with $\beta=0.2$

4.4 A Simple Example Case. Consider the same bilinear loading profile as in Eq. (32). The normalized effective crack length is shown to increase with the loading time in Fig. 10. The theoretical strength is reached as the effective crack length reaches the strip width. Figure 11 shows the normalized crack opening displacement versus the normalized loading time for the case of $\tau_{0} / t_{0}=0.5, \beta=0.33$, and $W / l_{\mathrm{ft}}=1.83$. The crack opening displacement is found to increase monotonously and nonlinearly with the loading time. It is seen that even when the external loading reaches the theoretical strength, the crack opening displacement is still smaller than the effective interaction distance $\delta_{c}$, indicating the strip is flaw tolerant under this loading rate.

Figure 12 plots the normalized critical strip width for the crack growth $W_{\mathrm{cr}} / l_{\mathrm{ft}}$ as a function of the normalized crack size $\beta$ under different loading rates. For a given loading rate, there always exists a minimum width indicated by the horizontal dashed line labeled as $W_{\mathrm{ft}}$, which is the flaw tolerant width below which cracks of all sizes are tolerated. The finding that the flaw-tolerance length scale increases with the loading rate suggests an additional protection mechanism of biological materials against impact loading: a viscoelastic material may be more robust against catastrophic failure at rapid loading rates. This is also consistent with the hypothesis that viscoelasticity could protect bone from dynamic loads (impact and vibration) by dissipating the parts of mechanical

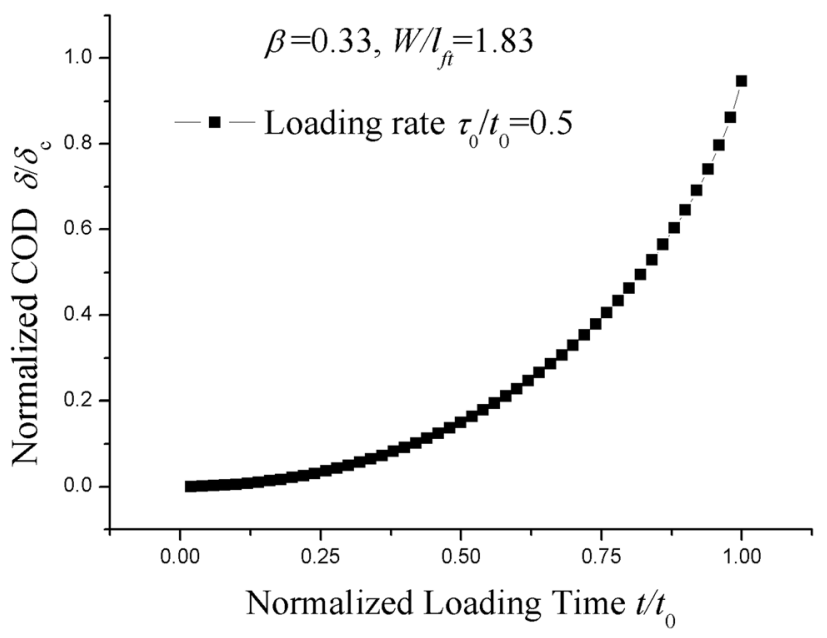

Fig. 11 The normalized crack opening displacement as a function of the loading time in the viscoelastic Dugdale problem with $\beta=0.33$ and $W / I_{\mathrm{ft}}=1.83$

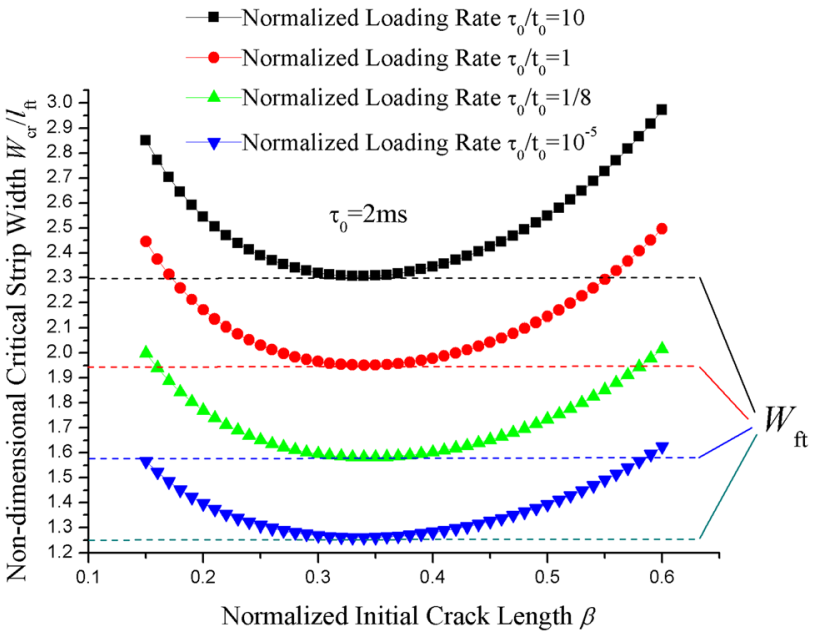

Fig. 12 The nondimensional critical strip width $W_{\mathrm{cr}} / I_{\mathrm{ft}}$ for crack growth as a function of the normalized crack length $\beta=a / W$ in the viscoelastic Dugdale problem under different loading rates

energy that could otherwise contribute to structural damage processes [38]. It can also be seen from Fig. 13 that, for very high or low loading rates, the flaw tolerant width approaches the corresponding elastic solution with the instantaneous or long-time Young's modulus, respectively. The result is consistent with the numerical analysis of flaw tolerant adhesion between a viscoelastic cylinder and a rigid substrate [31].

\section{Discussion}

The most elementary level of hierarchical structures of loadbearing biological materials such as bone exhibits a generic structure on the nanometer length scale. At the lowest level of bone organization, type-I collagen $(\sim 1.5 \mathrm{~nm}$ in diameter $)$ is assembled into fibrils (up to $15 \mu \mathrm{m}$ in length and $50-70 \mathrm{~nm}$ in diameter), and apatite crystals, $50 \times 25 \mathrm{~nm}$ in length and width and $2-3 \mathrm{~nm}$ in thickness, are nucleated at specific regions on or within the collagen fibrils [38]. Previous studies have been devoted to investigating why the elementary structure of biocomposites $[1,26,27,46]$ along with a superhard nanocrystalline coating [47] is generally organized at the nanometer length scale. A central hypothesis adopted in these studies is that the load-bearing biological

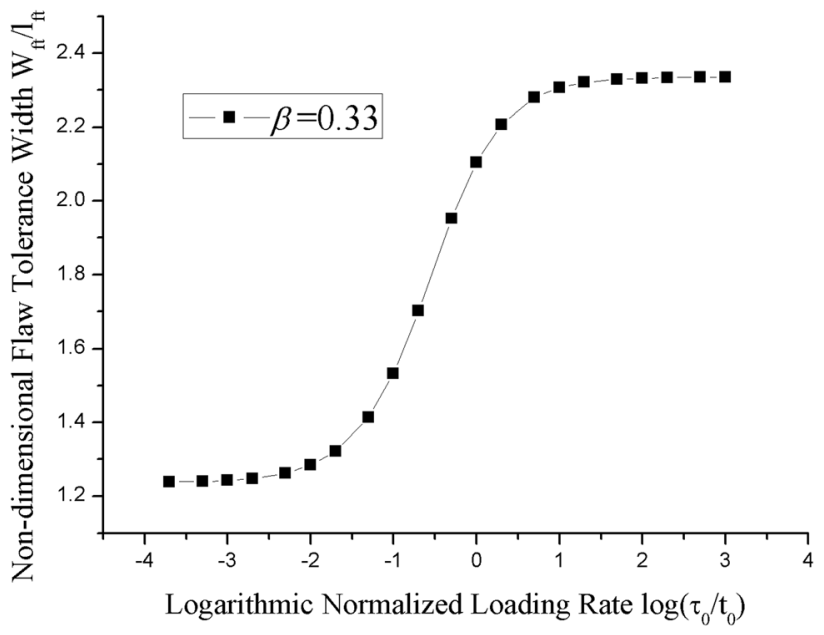

Fig. 13 The nondimensional flaw tolerance width $W_{\mathrm{ft}} / I_{\mathrm{ft}}$ as a function of the total loading time (different loading rates) with $\beta=0.33$ 
materials have been evolved to tolerate cracklike flaws at multiple size scales. The optimal state of a material which induces the maximum strength corresponds to a uniform distribution of stress at failure, even in the presence of cracklike flaws. In this state, the material fails by uniform rupture, rather than by crack propagation. This optimal state can be achieved simply by a size reduction $[1,6,8]$. Most of the previous studies have been restricted to purely elastic structures. However, as emphasized by Gao [8], the mechanical properties of bone and bonelike biomaterials should be time-dependent and Lakes and Katz [48] have analyzed different physical processes that are responsible for the viscoelasticity behaviors of bone. Sasaki et al. [49] showed that the viscoelasticity of bone can be attributed to its protein constituents. Inside the polymeric network of many types of protein, the statistical binding and rupture of cross links result in a small long-time modulus but a relatively stiff instantaneous response [26].

Bone, as an organic-inorganic composite, is generally able to maintain the stiffness of mineral platelets despite its soft viscoelastic protein constituents. This stiffness paradox has been explained by a tension-shear chain model of a mineral-protein composite in which the mineral platelets sustain the tensile load while protein transfers the load between the platelets via shear deformation [26]. The strength of the biocomposite thus hinges upon the strength of the mineral platelets; the latter was shown, using the virtual internal bond method [50], to be maximized and insensitive to flaws by their nanoscale size dimension $[1,26]$. Protein molecules can undergo large deformations as their domains unfold during stretching [51] and are thus naturally engineered with long ranges of deformation that can dramatically enhance the fracture toughness of the material. Slipping along the protein-mineral interface can further increase the effective strain to failure. On the contrary, the effective stress in the material is determined by the lower bound of the protein strength, interfacial strength, and mineral strength. A key to increasing the first two has been attributed to $\mathrm{Ca}^{2+}$ induced sacrificial bonds in bone [52]. The viscoelasticity of protein can also help biocomposites dissipate fracture energy under dynamic loads. For example, de Gennes [53] showed that the ratio between short-time and long-time elastic moduli plays an important factor in viscoelastic fracture energy dissipation.

In spite of the aforementioned progress, so far there are still no viscoelastic models available to understand flaw tolerance in ratesensitive materials. In the present paper, fracture mechanics concepts have been used to investigate the flaw tolerance of a center-cracked viscoelastic strip based on the Griffith and Dugdale models of crack growth. The classical correspondence principle is used in the case of Griffith's model and Graham's extended correspondence principle $[44,45]$ is adopted in the case of Dugdale's model. For a given crack size, analytical solutions to the critical strip width for crack growth, below which the tensile failure of the strip would not be induced by crack propagation but by the uniform rupture of material, is obtained for both models. In contrast to the elastic case, the critical width of the viscoelastic strip for crack growth is found to increase with the applied loading rate.

The flaw tolerant width, defined as the minimum critical strip width below which cracks of all sizes are tolerated, is found to increase with the loading rate and, in the cases of very high or low loading rates, coincides with the corresponding elastic solutions with an instantaneous or a long-time elastic modulus. This agrees with the previous numerical analysis by Chen et al. [31] on the flaw tolerance of an adhesive interface between a viscoelastic cylinder and a rigid substrate.

The flaw tolerant size of an elastic structure is usually on the nanometer length scale. The question of how this concept can be extended to macroscopic scales through structural hierarchy has been discussed by Gao [8]. The present analysis suggests that the loading rate could also be utilized to switch the material between flaw tolerance and flaw sensitive states. It should be interesting and challenging to further study the behaviors of hierarchical structures of rate-sensitive materials such as bone.
Furthermore, the present analysis should be useful for the design of new implant materials. It is well known that biomaterials need to fulfill a series of requirements. Besides interfacial and biological compatibility, the mechanical properties of an implant should match, as closely as possible, those of the tissue in contact. Besides the quasi-static mechanical properties, the need for an implant material to have similar viscoelastic properties to the tissue is, obviously, important. This means, for example, that an implant in contact should follow the same time-dependent behavior as the tissue when subjected to the same stress/strain history. This is a major reason why the viscoelastic properties of new materials or devices for medical applications should be carefully evaluated [38]. Since bone is a typically hierarchical and viscoelastic material, it remains a challenge to develop robust implants with similar mechanical properties as the natural bone.

\section{Acknowledgment}

This work is supported by the National Science Foundation of China (Grant Nos.10972220, 11125211, and 11021262) and the Project No. 2012CB937500. The authors would like to thank two anonymous reviewers for their helpful comments and suggestions.

\section{Appendix: Approximate Solutions to Crack Opening Displacement and Cohesive Zone Length in a Cracked Elastic Strip}

For a finite Dugdale crack in an infinite space shown in Fig. 14, the tractions on the crack face are divided into two regions

$$
\sigma(x)= \begin{cases}-\sigma_{\infty}, & |x|<a \\ \sigma_{s}-\sigma_{\infty}, & a<|x|<c\end{cases}
$$

In order to find the crack opening displacement, the classical distributed dislocation density method [54] can be used. For a dislocation density $f(x)$ over $-c<x<c$, we have the relation

$$
A \int_{-c}^{c} \frac{f(\xi) d \xi}{x-\xi}=\sigma(x), \quad|x|<c
$$

where $A=b \kappa E / 4 \pi$ and $b$ is the Burgers vector. Equation (A2) can be inverted as

$$
f(x)=-\frac{1}{\pi^{2} A \sqrt{x^{2}-c^{2}}} \int_{-c}^{c} \frac{\sqrt{\xi^{2}-c^{2}} \sigma(\xi) d \xi}{x-\xi}, \quad|x|<c
$$

which can be further rewritten as

$$
\begin{aligned}
f(x)= & -\frac{\sqrt{x^{2}-c^{2}}}{\pi^{2} A} \int_{-c}^{c} \frac{\sigma(\xi) d \xi}{(x-\xi) \sqrt{\xi^{2}-c^{2}}} \\
& +\frac{1}{\pi^{2} A \sqrt{x^{2}-c^{2}}} \int_{-c}^{c} \frac{\xi \sigma(\xi) d \xi}{\sqrt{\xi^{2}-c^{2}}}+\frac{x}{\pi^{2} A \sqrt{x^{2}-c^{2}}} \int_{-c}^{c} \frac{\sigma(\xi) d \xi}{\sqrt{\xi^{2}-c^{2}}}
\end{aligned}
$$

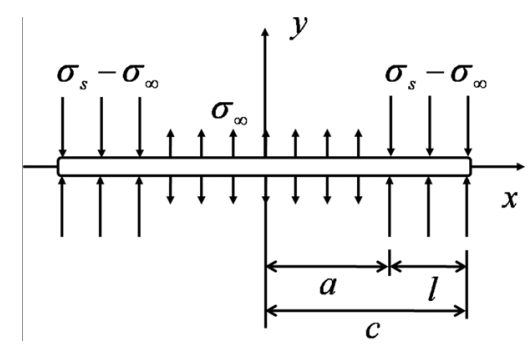

Fig. 14 Dugdale model of a finite crack in an infinite space 
When $\sigma(\xi)$ is an even function of $\xi$ and $\left.f(x)\right|_{x= \pm c}=0$, the second and third terms in the preceding equation vanish so that

$$
f(x)=-\frac{\sqrt{x^{2}-c^{2}}}{\pi^{2} A} \int_{-c}^{c} \frac{\sigma(\xi) d \xi}{(x-\xi) \sqrt{\xi^{2}-c^{2}}}
$$

The crack opening displacement is found by integrating the dislocation density from $c$ to $a$

$$
\delta=b N=b \int_{c}^{a} f(x) d x=\frac{2 b \sigma_{s} a}{\pi^{2} A} \ln \frac{c}{a}=\frac{8 \sigma_{s} a}{\pi \kappa E} \ln \frac{c}{a}
$$

Meanwhile, the requirement that the third term on the right side of Eq. (A4) should vanish leads to

$$
\int_{-c}^{c} \frac{\sigma(\xi) d \xi}{\sqrt{\xi^{2}-c^{2}}}=0
$$

from which the length of the cohesive zone ahead of the crack tip can be deduced as

$$
-\frac{\pi}{2} \sigma_{\infty}+\sigma_{s} \arccos \frac{a}{a+l}=0
$$

For a finite-width strip model with a center crack, the solution to a periodic array of cracks can be adopted to approximate the finite width problem, as shown in Fig. 15. In this case, we have

$A \sum_{m=-\infty}^{+\infty} \int_{-c}^{c} \frac{f(\lambda) d \lambda}{x-\lambda-2 m W}=\sigma(x) \quad-c+2 m W<x<c+2 m W$

Using the following equation

$$
\sum_{m=-\infty}^{+\infty} \frac{1}{x-\lambda-2 m W}=\cot \frac{\pi(x-\lambda)}{2 W}
$$

Eq. (A9) can be simplified as

$$
\frac{\pi}{2 W} \int_{-c}^{c} \frac{\cos \frac{\pi \lambda}{2 W} f(\lambda) d \lambda}{\sin \frac{\pi x}{2 W}-\sin \frac{\pi \lambda}{2 W}}=\frac{\sigma(x)}{A}
$$

Using the following variable transformations

$$
\lambda^{\prime}=\sin \frac{\pi \lambda}{2 W}, \quad x^{\prime}=\sin \frac{\pi x}{2 W}
$$

Eq. (A11) becomes

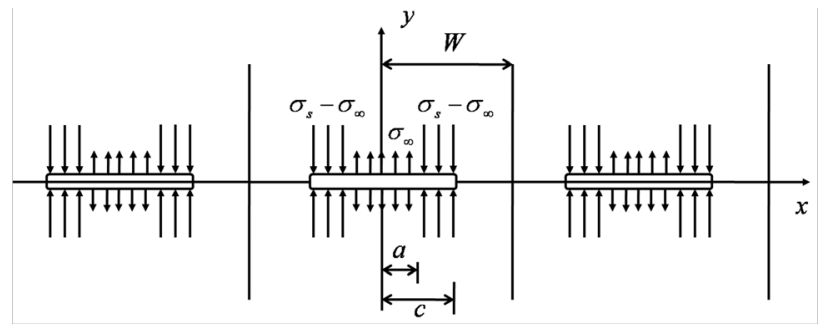

Fig. 15 Dugdale model of a periodic array of cracks in an infinite space. Each crack has a length $a$ and a cohesive zone length $c-a$. The length of the period is $2 W$.

$$
\int_{-c^{\prime}}^{c^{\prime}} \frac{M\left(\lambda^{\prime}\right) d \lambda^{\prime}}{x^{\prime}-\lambda^{\prime}}=\frac{\sigma^{\prime}\left(x^{\prime}\right)}{A}
$$

where $M\left(\lambda^{\prime}\right)=f(\lambda)$ and $\sigma^{\prime}\left(x^{\prime}\right)=\sigma(x)$.

Since Eq. (A13) has the same form as Eq. (A1), the solution can be written as

$$
\begin{aligned}
& M\left(x^{\prime}\right)=-\frac{1}{\pi^{2} A \sqrt{x^{\prime 2}-c^{\prime 2}}} \int_{-c}^{c} \frac{\sqrt{\lambda^{\prime 2}-c^{\prime 2}} \sigma^{\prime}\left(\lambda^{\prime}\right) d \lambda^{\prime}}{x^{\prime}-\lambda^{\prime}}, \\
& -c+2 m H<\left|x^{\prime}\right|<c+2 m H
\end{aligned}
$$

where $c^{\prime}=\sin (\pi c / 2 W)$.

In a manner similar to the single crack case, the crack opening displacement is

$$
\delta=\frac{8 \sigma_{s} W}{\pi \kappa E} I(c)
$$

where

$I(c)=\int_{\theta}^{\pi / 2} \frac{c^{\prime} \cos X}{\pi \sqrt{1-c^{\prime 2} \sin ^{2} X}} \ln \frac{(\sin \theta-\sin X)(1-\cos (\theta+X))}{(\sin \theta+\sin X)(1-\cos (\theta-X))} d X$

Here, $\sin X=x^{\prime} / c^{\prime}, \sin \theta=a^{\prime} / c^{\prime}$, and $a^{\prime}=\sin (\pi a / 2 W)$.

The length of the cohesive zone in the finite-width strip model

$$
-\frac{\pi}{2} \sigma_{\infty}+\sigma_{s} \arccos \frac{a^{\prime}}{c^{\prime}}=0
$$

which can be rewritten in the form

$$
\alpha=\frac{c}{W}=\frac{2}{\pi} \arcsin \frac{\sin \frac{\pi \beta}{2}}{\cos \frac{\pi \sigma_{\infty}}{2 \sigma_{s}}}
$$

\section{References}

[1] Gao, H., Ji, B., Jäger, I. L., Arzt, E., and Fratzl, P., 2003, "Materials Become Insensitive to Flaws at Nanoscale: Lessons From Nature," Proc. Natl. Acad. Sci. U.S.A., 100, pp. 5597-5600.

[2] Persson, B. N. J., 2003, "Nanoadhesion," Wear, 254, pp. 832-834.

[3] Glassmaker, N. J., Jagota, A., Hui, C. Y., and Kim, J., 2004, "Design of Biomimetic Fibrillar Interface: 1. Making Contact,” J. R. Soc. Interface, 1, pp 23-33.

[4] Hui, C. Y., Glassmaker, N. J., Tang, T., and Jagota, A., 2004, "Design of Biomimetic Fibrillar Interface: 2. Mechanics of Enhanced Adhesion,” J. R. Soc. Interface, 1, pp. 35-48.

[5] Gao, H., Wang, X., Yao, H., Gorb, S., and Arzt, E., 2005, "Mechanics of Hierarchical Adhesion Structures of Geckos," Mech. Mater., 37, pp. 275-285.

[6] Gao, H., and Chen, S., 2005, "Flaw Tolerance in a Thin Strip Under Tension," ASME J. Appl. Mech., 72, pp. 732-737.

[7] Northen, M. T., and Turner, K. L., 2005, "A Batch Fabricated Biomimetic Dry Adhesive," Nanotechnology, 16, pp. 1159-1166.

[8] Gao, H., 2006, "Application of Fracture Mechanics Concepts to Hierarchical Biomechanics of Bone and Bone-Like Materials," Int. J. Fract., 138, pp. 101-137.

[9] Dugdale, D. S., 1960, "Yielding of Steel Sheets Containing Slits," J. Mech. Phys. Solids, 8(2), pp. 100-104.

[10] Bilby, B. A., Cottrell, A. H., and Swinden, K. H., 1963, "The Spread of Plastic Yield From a Notch," Proc. R. Soc. London, Ser. A, 272, pp. 304-314.

[11] Bazant, Z. P., 1976, "Instability, Ductility and Size Effect in Strain-Softening Concrete," J. Eng. Mech., 102, pp. 331-344.

[12] Hillerborg, A., Modeer, M., and Petersson, P. E., 1976, "Analysis of Crack Formation and Crack Growth in Concrete by Means of Fracture Mechanics and Finite Elements," Cem. Concr. Res., 6, pp. 773-782.

[13] Kendall, K., 1978, "Complexities of Compression Failure," Proc. R. Soc. London, Ser. A, 361, pp. 245-263.

[14] Karihaloo, B. L., 1979, "A Note on Complexities of Compression Failure," Proc. R. Soc. London, Ser. A, 368, pp. 483-493.

[15] Rice, J. R., 1980, "The Mechanics of Earthquake Rupture," International School of Physics "E. Fermi," Course 78, 1979, Italian Physical Society/North Holland, Amsterdam. 
[16] Bazant, Z. P., and Cedolin, L., 1983, "Finite Element Modeling of Crack Band Propagation,” J. Struct. Eng., 109, pp. 69-92.

[17] Barenblatt, G. I., 1985, "The Mathematical Theory of Equilibrium Cracks in Brittle Fracture,” Adv. Appl. Mech., 7, pp. 55-129.

[18] Bao, G., and Suo, Z., 1992, "Remarks on Crack-Bridging Concepts," Appl. Mech. Rev., 45, pp. 355-366.

[19] Cox, B. N., and Marshall, D. B., 1994, "Concepts for Bridged Cracks in Fracture and Fatigue," Acta Metall. Mater., 42, pp. 341-363.

[20] Bazant, Z. P., and Planas, J., 1998, Fracture and Size Effect in Concrete and Other Quasibrittle Materials, CRC, Boca Raton, FL.

[21] Massabo, R., and Cox, B. N., 1999, "Concepts for Bridged Mode II Delamination Cracks," J. Mech. Phys. Solids, 47, pp. 1265-1300.

[22] Mulmule, S. V., and Dempsey, J. P., 2000, "LEFM Size Requirement for the Fracture Testing of Sea Ice," Int. J. Fract., 102, pp. 85-98.

[23] Drugan, W. J., 2001, "Dynamic Fragmentation of Brittle Materials: Analytical Mechanics-Based Models,” J. Mech. Phys. Solids, 49, pp. 1181-1208.

[24] Gao, H., Ji, B., Buehler, M. J., and Yao, H., 2004, "Flaw Tolerant Bulk and Surface Nanostructures of Biological Systems," Mech. Chem. Biosyst., 1(1), pp. 37-52.

[25] Gao, H., and Yao, H., 2004, "Shape Insensitive Optimal Adhesion of Nanoscale Fibrillar Structures," Proc. Natl. Acad. Sci. U.S.A., 101, pp. 7851-7856.

[26] Ji, B., and Gao, H., 2004, "Mechanical Properties of Nanostructure of Biological Materials," J. Mech. Phys. Solids, 52, pp. 1963-1990.

[27] Ji, B., and Gao, H., 2004, "A Study of Fracture Mechanisms in Biological Nano-Composites via the Virtual Internal Bond Model," Mater. Sci. Eng. A, 366, pp. 96-103.

[28] Buehler, M. J., Yao, H., Ji, B., and Gao, H., 2006, "Cracking and Adhesion at Small Scales: Atomistic and Continuum Studies of Flaw Tolerant Nanostructures," Modell. Simul. Mater. Sci. Eng., 14, pp. 799-816.

[29] Yao, H., and Gao, H., 2006, "Mechanics of Robust and Releasable Adhesion in Biology: Bottom-Up Designed Hierarchical Structures of Gecko," J. Mech. Phys. Solids, 54, pp. 1120-1146.

[30] Chen, S., and Soh, A., 2008, "Tuning the Geometrical Parameters of Biomimetic Fibrillar Structures to Enhance Adhesion," J. R. Soc. Interface, 5, pp. 373-382.

[31] Chen, S., Xu., G., and Soh, A., 2010, "Size-Dependent Adhesion Strength of a Single Viscoelastic Fiber," Tribol. Lett., 37, pp. 375-379.

[32] Chen, S., and Chen, P., 2010, "Nanoadhesion of a Power-Law Graded Elastic Material," Chin. Phys. Lett., 27(10), p. 108102.

[33] Kumar, S., Haque, M. A., and Gao, H., 2009, "Notch Insensitive Fracture in Nanoscale Thin Films," Appl. Phys. Lett., 94, p. 253103.

[34] Kumar, S., Li, X. Y., Haque, A., and Gao, H. J., 2011, "Is Stress Concentration Relevant for Nanocrystalline Metals?,"Nano Lett., 11, pp. 2510-2516.

[35] Giesa, T., Arslan, M., Pugno, N. M., and Buehler, M. J., 2011, "Nanoconfinement of Spider Silk Fibrils Begets Superior Strength, Extensibility, and Toughness," Nano Lett., 11(11), pp. 5038-5046.

[36] Abou Neel, E. A., Salih, V., Revell, P. A., and Young, A. M., 2012, "Viscoelastic and Biological Performance of Low-Modulus, Reactive Calcium
Phosphate-Filled, Degradable, Polymetric Bone Adhesive," Acta Biomater., 8 , pp. 313-320.

[37] Deymier-Black, A. C., Yuan, F., Singhal, A., Almer, J. D., Brinson, L. C., and Dunand, D. C., 2012, "Evolution of Load Transfer Between Hydroxyapatite and Collagen During Creep Deformation of Bone," Acta Biomater., 8, pp. 253-261.

[38] Mano, J. F., 2005, "Viscoelastic Properties of Bone: Mechanical Spectroscopy Studies on a Chicken Model," Mater. Sci. Eng., C, 25, pp. 145-152.

[39] Fung, Y. C., 1983, Biomechanics, Science Press, Beijing (in Chinese).

[40] Nuismer, R. J., 1974, "Governing Equation for Quasi-Static Crack Growth in Linearly Viscoelastic Materials,” ASME J. Appl. Mech., 41, pp. 631-634.

[41] Gurtin, M. E., and Sternberg, E., 1962, "On the Linear Theory of Viscoelasticity,” Arch. Ration. Mech. Anal., 11, pp. 291-356.

[42] Christensen, M. E., 1982, Theory of Viscoelasticity, Academic, New York.

[43] Gao, Q., Lin, S., and Yang, X. J., 2007, "Description of Non-Linear Creep Constitutive Relation for Viscoelastic Butyl Rubber," Chin. J. Appl. Mech., 24, pp. 386-390 (in Chinese).

[44] Graham, G. A. C., 1968, "Correspondence Principle of Linear Viscoelasticity Theory for Mixed Boundary Value Problems Involving Time-Dependent Boundary Regions," Q. Appl. Math., 26, pp. 167-174.

[45] Graham, G. A. C., and Sabin, G. C. W., 1973, "Correspondence Principle of Linear Viscoelasticity for Problems That Involve Time-Dependent Regions," Int. J. Eng. Sci., 11, pp. 123-140.

[46] Guo, X., and Gao, H., 2005 "Bio-Inspired Material Design and Optimization," IUTAM Symposium on Topological Design Optimization of Structures, Machines and Materials-Status and Perspectives, Rungstedgaard, Copenhagen, Denmark, October 26-29.

[47] Kauffmann, F., Ji, B., Dehm, G., Gao, H., and Arzt, E., 2005, "A Quantitative Study of the Hardness in a Superhard Nanocrystalline Titanium Nitride/Silicon Nitride Coating," Scr. Mater., 52, pp. 1269-1274.

[48] Lakes, R. S., and Katz, J. L., 1979, "Viscoelastic Properties of Wet Cortical Bone-II: Relaxation Mechanisms," J. Biomech., 12(9), pp. 679-687.

[49] Sasaki, N., Nakayama, Y., Yoshikawa, M., and Enyo, A., 1993, "Stress Relaxation Function of Bone Collagen," J. Biomech., 26(12), pp. 1369-1376.

[50] Gao, H., and Klein, P. 1998, "Numerical Simulation of Crack Growth in an Isotropic Solid With Randomized Internal Cohesive Bonds," J. Mech. Phys. Solids, 46, pp. 187-218.

[51] Smith, B. L., Schaeffer, T. E., Viani, M., Thompson, J. B., Frederick, N. A., Kindt, J. H., Belcher, A., Stucky, G. D., Morse, D. E., and Hansma, P. K., 1999 "Molecular Mechanistic Origin of the Toughness of Natural Adhesive, Fibres and Composites," Nature (London), 399, pp. 761-763.

[52] Thompson, J. B., Kindt, J. H., Drake, B., Hansma, H. G., Morse, D. E., and Hansma, P. K., "Bone Indentation Recovery Time Correlates With Bone Reforming Time," Nature (London), 414, pp. 773-776.

[53] de Gennes, P. G., 1996, "Soft Adhesives," Langmuir, 12, pp. 4497-4500.

[54] Bilby, B. A., and Eshelby J. D., 1968, Fracture, Academic, New York. 